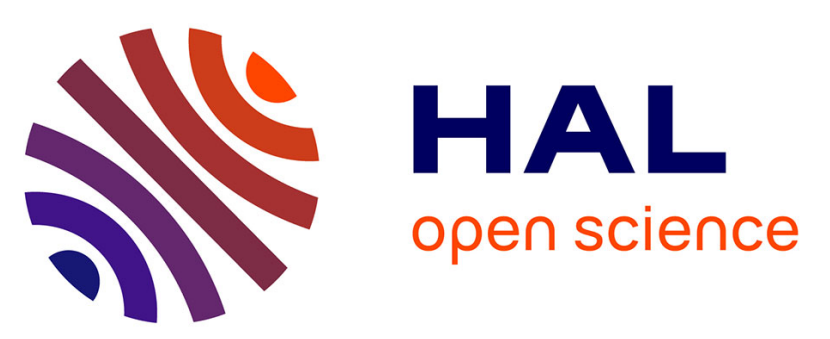

\title{
Chemical communication and dynamics of droplet emulsions in networks of Belousov-Zhabotinsky micro-oscillators produced by microfluidics
}

Kristian Torbensen, Federico Rossi, Sandra Ristori, Ali Abou-Hassan

\section{- To cite this version:}

Kristian Torbensen, Federico Rossi, Sandra Ristori, Ali Abou-Hassan. Chemical communication and dynamics of droplet emulsions in networks of Belousov-Zhabotinsky micro-oscillators produced by microfluidics. Lab on a Chip, 2017, 10.1039/C6LC01583B . hal-01480126

\section{HAL Id: hal-01480126 \\ https: / hal.sorbonne-universite.fr/hal-01480126}

Submitted on 1 Mar 2017

HAL is a multi-disciplinary open access archive for the deposit and dissemination of scientific research documents, whether they are published or not. The documents may come from teaching and research institutions in France or abroad, or from public or private research centers.
L'archive ouverte pluridisciplinaire HAL, est destinée au dépôt et à la diffusion de documents scientifiques de niveau recherche, publiés ou non, émanant des établissements d'enseignement et de recherche français ou étrangers, des laboratoires publics ou privés. 


\title{
Chemical communication and dynamics of droplet emulsions in networks of Belousov-Zhabotinsky micro-oscillators produced by microfluidics
}

\begin{abstract}
Kristian Torbensen, ${ }^{a \dagger}$ Federico Rossi, ${ }^{b+}$ Sandra Ristori, ${ }^{\mathrm{c}}$ and Ali Abou-Hassan ${ }^{\mathrm{a} *}$
Chemical communication leading to synchronization and collective behaviour of dynamic elements, such as cell colonies, is a widespread phenomenon with biological, physical and chemical importance. Such synchronization between elements proceeds via chemical communication by emmision, interdiffusion and reception of specific messenger molecules. On a lab scale, these phenomena can be modeled by encapsulating an oscillating chemical reaction, which serves as a signal (information) sender/receiver element, inside microcompartments such as droplet emulsions, liposomes and polymersomes. Droplets can thus be regarded as single units, able to generate chemical messengers that diffuse in the environment and hence can interact with other compartments. The Belousov-Zhabotinsky (BZ) reaction is a well-known chemical oscillator largely used as a model for complex nonlinear phenomena, including chemical, physical and biological examples. When the BZ-reaction is encapsulated inside microcompartments, its chemical intermediates can serve as messengers by diffusing among different microcompartments, to trigger specific reactions leading to a collective behavior between the elements. The geometry and constitution of the diffusion pathwaysplays here an important role in governing the collective behaviour of the system. In this context, microfluidics is not only a a versatile tool for mastering the encapsulation process of the BZ-reaction in monodisperse microcompartments but also for creating geometries and newtorks with well defined boundaries... The individual compartments can be engineered with selected properties using different surfactants in the case of simple emulsions, or with specific membrane properties in the case of liposomes. Furthermore, it enables the arrangment of these microcompartments in various geometric configurations, where the diffusive coupling pathways between individual compartments are both spatially and chemically well-defined. In this tutorial paper, we review a number of articles reporting various approaches to generate networks of compartmentalized Belousov-Zhabotinsky (BZ) chemical oscillators using microfluidics. In contrast to biological cellular networks, the dynamical characteristics of the BZ-reaction is well-known and, when confined in microcompartments arranged in different configurations with a pure interdiffusive coupling, these communicative microreactors can serve to mimic various types of bio-physical networks, aiding to comprehend the concept of chemical communication.
\end{abstract}

\section{Introduction}

The generation, propagation and reception of bio/chemical information between individual organisms are the keystones of many intelligent communicating systems. ${ }^{1}$ The transmission of a signal at the level of an individual generator-receptor couple is for example the basis for the bio/chemical signal transmission in neural synaptic communication. ${ }^{2,3}$ The propagation of a signal wave-front among different individual sources is then associated with the synchronization of the sources and the observation of cooperative phenomena. ${ }^{4}$ Such cooperative effects are ubiquitous in nature. They are observed for example in the synchronous flashing within

\footnotetext{
a. Sorbonne Universités, UPMC Univ Paris 06, UMR 8234, Laboratoire PHysicochimie des Electrolytes, Nanosystèmes InterfaciauX (PHENIX), 4 place Jussieu case 51, 75252 Paris cedex 05 - France

${ }^{b .}$ Department of Chemistry and Biology, University of Salerno, Via Giovanni Paolo II 132, Fisciano (SA), Italy

Department of Earth Sciences \& CSGI, University of Florence, Via della Lastruccia

3, 50019 Sesto Fiorentino, Firenze, Italy

d. Authors contributed equally
}

colonies of fireflies, in the contraction/expansion of heart muscles, in morphogenesis, calcium signalling dynamics, decentralized bacterial quorum sensing, etc. ${ }^{1,2}$ Specifically, in cell populations, a variety of dynamical behaviours have been reported, ranging from a single synchronization state to multiple synchronization states, which reflect different degrees of communication..$^{5}$ Among the mechanisms through which communication is achieved, the most characteristics for living systems are the (frequently negative, but sometime positive) feedback loops, closely related to the oscillation phenomena described in this review. 6,7

At the extremities, synchronization in nature occurs, on the smallest scale, as simultaneously moving electrons in superconductors, and at the largest scale, as planetary systems hurled by gravitational synchrony. ${ }^{8}$ At the level of unicellular organisms, cooperation and synchronization occurs via chemical communication based on a messenger diffusion/reaction, which extends over a wide range of time and length-scales. ${ }^{9,} 10$ Inside a cell, within trans-membrane protein machinery, chemical communication is restricted to the nanometer scale. However, in many other examples, chemical reagents are able to cross biological membranes and be transported to their target by diffusion over larger distances in an aqueous environment. For instance, the 
nervous system involves micron-scale chemical communication during synaptic communication from neurons to axons. ${ }^{11}$ Diffusion of chemical reagents over even longer distances (hundreds of micrometers or more) in the extracellular solution is believed to be responsible of the largescale collective behaviours of colonies of unicellular organisms. For trans-membrane communication over short or large distance a key role can also be assigned to specific lipids. For instance, phosphatidylinositol derivatives have been charged to promote cell migration by generating spontaneous waves which results in oscillatory dynamics. This example points out the relevance of the membrane composition, not only in terms of biomacromolecules embedded in the lipid matrix but also in terms of the very same lipids forming the bilayer skeleton. ${ }^{12}$ Synchronized and cooperative behaviour are also widely exploited in our everyday life through information and communication technology (ICT). Inspired by biological paradigms, new technologies emerges, such as the bio/chemical-information and communication technology (bio/chemical-ICT), ${ }^{11,13,14}$ which aims at extending the wellknown field of ICT, classically based on the transmission of electrical or electromagnetic signals, to the bio/chemical world of molecules. ${ }^{15,16}$ In a biomimetic approach, it proposes to carry chemical information into individual encapsulated microchemical reactors, and to propagate chemical information between them. Such naturally occurring information processes can provide a basis for innovative computational strategies. ${ }^{14,15,17}$

Motivated by mimicking and understanding the collective behaviour and synchronicity involved in complex systems in living organisms, or by more applied objectives such as the field of ICT, the last ten years have witnessed extensive research in the field of chemical communication and dynamics of oscillatory droplets. To build reliable communicating systems capable of exhibiting dynamic and collective behaviour, several requirements must be fulfilled. First, the encapsulated chemical reaction should have a non-linear mechanism able to respond to external stimuli and, at the same time, generate chemical signals in a controllable way. The Belousov-Zhabotinsky (BZ) reaction, ${ }^{18,19}$ described in more detail in the following section, certainly matches these requisites. It is a well-known chemical oscillator, whose mechanism is effected by the interaction of activatory and inhibitory feedback loops, capable of generating periodic chemical signals. Second, once the signal emitter/receiver reaction is "stored" inside droplets, the interface of the droplets should be (semi) permeable to allow the propagation and transmission of the chemical information from one compartment to another, via chemical messengers, such as in living organisms. Various surfactants, phospholipids and polymers are usually employed to form such membrane-like interfaces. Third, there must be a reliable method of droplet generation capable of controlling the droplet size and dispersity, the chemical composition of the liquid/liquid interface between the droplets and the surrounding medium, as well as the spatial organization of the droplets. Here, microfluidic systems (vide infra) can offer unprecedented opportunities to control such parameters.

As a consequence, an ideal experimental reaction-diffusion system that has been largely investigated in order to study chemical communication and the resulting dynamics, consists of microcompartments (emulsions, liposomes, polymersomes) produced using microfluidics, in which aqueous droplets containing the Belousov-Zhabotinsky (BZ) chemical oscillator are dispersed in oil or aqueous media.

Although biological and biochemical oscillators exist, ${ }^{20-22}$ and can be used as a model of chemical information, interpretation of the autonomous and collective behaviour in such networks is onerous due to the complexity of the dynamics and the chemistry of the systems. In contrast, the chemistry of the BZreaction is well-understood; its rate constants are known, and the inter-droplet coupling between compartments is purely diffusive. $^{23}$

The aim of this manuscript is to provide a tutorial review to the field of chemical communication and dynamics in networks of compartments containing BZ micro-oscillators engineered using microfluidics. First the BZ-reaction is introduced, then the important role of microfluidics for generating communicative networks of micro-oscillators is discussed and finally different experimental efforts that have been achieved and reported recently in this field using microfluidics are reviewed

For a more theoretical description of communicative networks of diffusively coupled oscillators, the reader can turn to these reports. $^{24-29}$

\section{The Belousov-Zhabotinsky (BZ) chemical reaction as a system for chemical communication studies}

Nowadays, the BZ reaction is probably the most studied oscillating chemical reaction; as stated by Anatol Zhabotinsky; ${ }^{30}$ "the popularity of the BZ is mainly due to the fact that it is the simplest closed macroscopic system that can be maintained far from equilibrium by an internal source of free energy homogeneously distributed in space". This means that even in batch experiments, the BZ reaction can last for several hours without the need of supplying fresh reagents to the system; moreover, being outside of thermodynamic equilibrium, BZ can display several exotic dynamical regimes: periodic, aperiodic and chaotic oscillations, ${ }^{31}$ autocatalysis and bistability, ${ }^{32}$ Turing structures and pattern formation. ${ }^{33,34}$ For a more detailed review on the $\mathrm{BZ}$ reaction, we encourage the reader to consult these articles. ${ }^{32,35-38}$ Here, we briefly sketch the oscillatory mechanism, and highlight the most important features that make the BZ reaction a good system to be coupled with microfluidic for studying chemical communication.

The $\mathrm{BZ}$ reaction is driven by the oxidation of an organic substrate, generally malonic acid, by bromate in an acidic solution in the presence of a catalytic species. The most used catalysts are redox active organo-metal complexes such as ferroin (a phenanthroline-iron(II) complex) and tris(2,2'bipyridyl)-ruthenium or metal ions such the redox couple 
$\mathrm{Ce}^{4+} / \mathrm{Ce}^{3+}$ or $\mathrm{Mn}^{3+} / \mathrm{Mn}^{2+}$. An example of the oscillating behavior is given in Figure 1A, where a spectrophotometric time-series show the changes in the concentration of the reduced form of the catalyst. In the specific case of ferroin, the two different oxidation states are reflected in a dramatic change of color, blue when oxidized and red when reduced.

The simplest and oldest mechanism, which account for the oscillations of the BZ-system is known as FKN (proposed by Field, Korös and Noyes), ${ }^{39}$ sketched in Figure 1B with ferroin as the catalyst. The scheme briefly consists of three main processes with three key species responsible for the activatory and inhibitory feedback loops that compete in the different stages of the reaction, giving rise to a periodic change in the concentrations of the reaction intermediates. Process $A$ takes place when the concentration of bromide ions, $\left[\mathrm{Br}^{-}\right]$, an intermediate produced by the oxidation of the organic substrate during the early stages of the reaction, is above a certain critical threshold, dictated by the experimental condition, $\left[\mathrm{Br}^{-}\right]_{\mathrm{cr}}$. During process $\mathrm{A}$ bromide ions are consumed to yield bromous acid $\left(\mathrm{HBrO}_{2}\right)$, until $\left[\mathrm{Br}^{-}\right]$falls down below $\left[\mathrm{Br}^{-}\right.$ ]$_{\mathrm{cr}}$ and process $\mathrm{B}$ is initiated. During process $\mathrm{B}$ the autocatalytic species $\mathrm{HBrO}_{2}$ quickly oxidizes the reduced form of the catalyst, $\mathrm{Fe}$ (II), until a high concentration of $\mathrm{Fe}$ (III) initiates process (C); here the catalyst is reduced by the organic substrate to yield $\mathrm{Br}^{-}$and resume the cycle. The three processes can be described by the overall reactions:

Process A:

Process B:

$$
\mathrm{BrO}_{3}^{-}+5 \mathrm{Br}^{-}+6 \mathrm{H}^{+} \rightarrow 3 \mathrm{Br}_{2}+\mathrm{H}_{2} \mathrm{O}
$$

$\mathrm{BrO}_{3}^{-}+\mathrm{HBrO}_{2}+2 \mathrm{Fe}^{2+}+3 \mathrm{H}^{+} \rightarrow 2 \mathrm{HBrO}_{2}+2 \mathrm{Fe}^{3+}+\mathrm{H}_{2} \mathrm{O}$

Process C:

$$
2 \mathrm{Fe}^{3+}+\mathrm{MA}+\mathrm{BrMA} \rightarrow \mathrm{fBr}^{-}+2 \mathrm{Fe}^{2+}+\mathrm{P}
$$

Where MA stands for malonic acid, $\mathrm{CH}_{2}(\mathrm{COOH})_{2}$, BrMA for bromomalonic acid, $\mathrm{BrCH}(\mathrm{COOH})_{2}$, and $f$ is a stoichiometric factor which depends on the experimental conditions.

In the FKN model $\mathrm{Br}^{-}$acts as inhibitor of oscillations, bringing the system in a reduced state. On the contrary, species like $\mathrm{HBrO}_{2}$ and the catalyst promote oscillations and act as activators. Despite the relatively simple scheme, during each oscillatory cycle the BZ reaction produces several chemical intermediates having different physico-chemical properties (charge, hydrophobicity, etc.) and more species can be active in promoting $\left(\mathrm{Br}_{2} \mathrm{O}_{4}, \mathrm{BrO}_{2}{ }^{\circ}\right)$ or inhibiting $\left(\mathrm{Br}_{2}\right)$ the oscillations. Occasionally, Ru-based catalysts are employed to render the reaction photosensitive. The photo-excited state of the Ruthenium biprydine complex $\left(\lambda_{\max }=450 \mathrm{~nm}\right)$, for example, is able to interact with bromate and oxygen to promote the formation of $\mathrm{Br}^{-}$, thus acting as a powerful oscillations inhibitor, externally controllable by light. $^{40}$ This feature is particularly useful to set initial and boundary conditions of BZbased oscillator networks.

As previously stated, the BZ reaction can produce several oscillation cycles (over a thousand for certain experimental parameters) even in batch conditions, thus in typical chemical communication experiments, the $\mathrm{BZ}$ can be easily confined with no need of feeding with fresh reactants; together with the "long life", another aspect that makes BZ appealing for such kind of experiments is the variety of intermediates produced during each cycle and their influence on the oscillations. In fact, when single oscillators are physically separated by a barrier or by a solvent immiscible with water, the intermediates can be used as messengers to deliver chemical information among different single units. By exploiting the properties of the intermediates, it is possible to choose the barriers (or the separating solvent) in order to have a specific type of coupling (global, inhibiting or activating) and the corresponding feedback on the oscillating mechanisms.

\section{(A)}
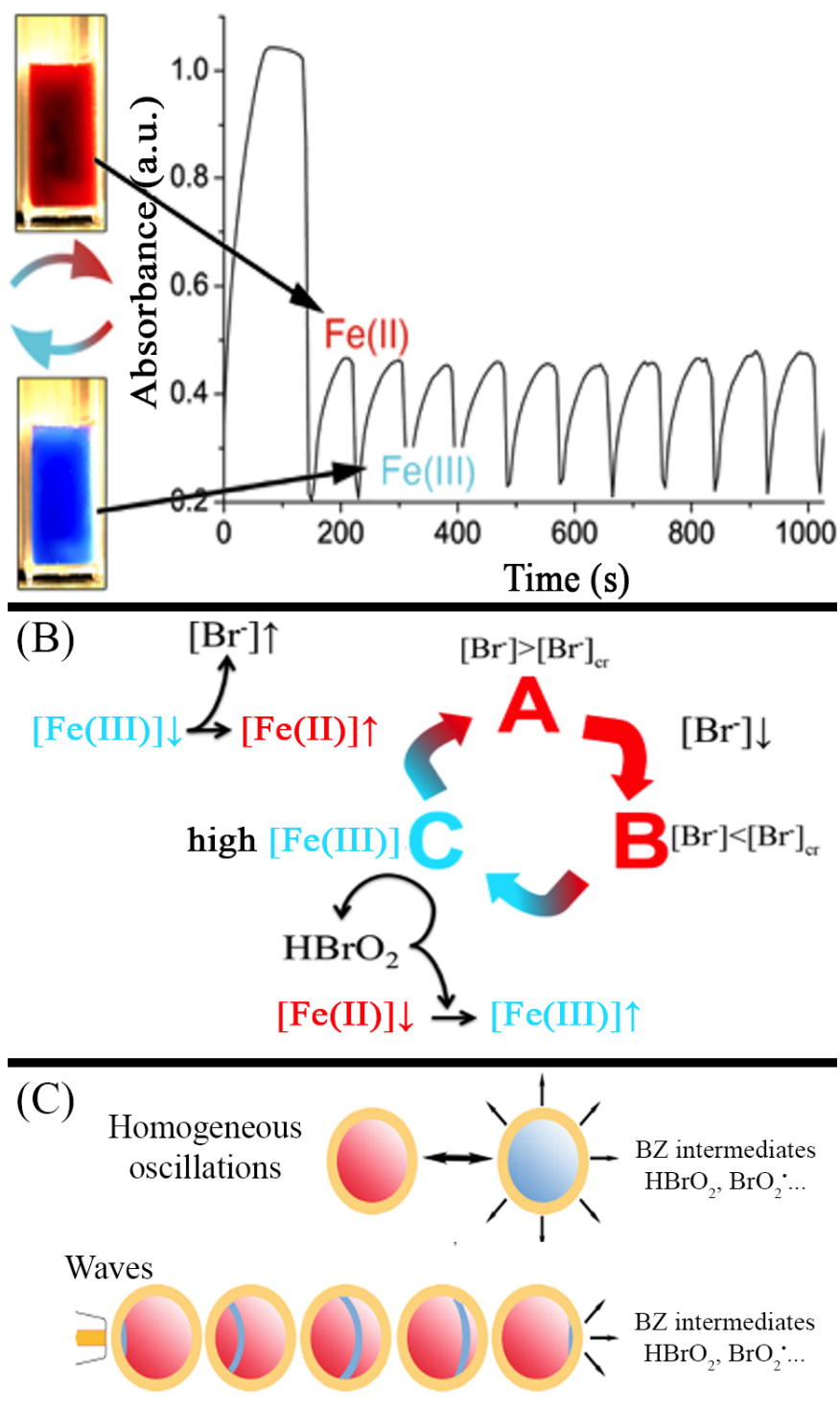

Figure 1. (A) Spectrophotometric recording at $\lambda=510 \mathrm{~nm}$ (where ferroin has an absorption maximum) of a typical BZ reaction with malonic acid as organic substrate. Pictures on the left show the catalyst colour in the different oxidation states; (B) simplified sketch of the FKN mechanism. Different colours refer to the catalyst oxidation state during each stage of the reaction; (C) Signals directionality related with the type of dynamical behaviour of the BZ reaction. 
The compartmentalization of the BZ reaction in nano- and microreactors, microparticles, vesicles, liposomes, polymersomes, or aqueous microdroplets, has been used as a model system to investigate short and long-range chemical communication between microreactors arranged in various configurations, from linear arrays to network with intricate geometries and multiple connections. ${ }^{9,41-56}$

An important issue for controlling the communication dynamics in networks of chemical oscillators is the way the signal leaves one unit to reach the others. For example, messenger molecules can target a specific area or they can uniformly spread in the surrounding environment, depending on the dynamical behavior of the chemical oscillator inside a single compartment (see Figure 1C). One of the crucial parameter influencing the global dynamics of the $B Z$ reaction is the medium homogeneity; in particular, in the absence of any macroscopic concentration gradients, the system oscillates uniformly in space (homogeneous oscillations). On the contrary, under the action of spatial gradients, the BZ behaves as a reaction-diffusion system generating fronts, pulses or waves. ${ }^{57-59}$ When the $\mathrm{BZ}$ system is confined and no stirring or advection is possible, the critical parameter responsible for the homogeneity of the system is the compartment size. In the case of droplets, the diameter $d$ determines the time of diffusive mixing, $t=d^{2} / 2 D$ (with $D$ being the diffusion coefficient of a BZ intermediate), that has to be compared with the $B Z$ oscillation period, $\tau$ : when $t<\tau$, bulk oscillations generate an isotropic distribution of the BZ intermediates in the inner aqueous phase, which, in turn, may cross the amphiphilic barriers in each point of the membrane; when $t>$ $\tau$, the coupling of the BZ with diffusion generates pulses or chemical waves, which are directional signals propagating along a straight line and can be controlled to deliver information to a precise target. Therefore, by taking advantage of the microfluidic techniques, it is relative simple to adjust the size of the compartments to obtain different ways of signal delivery.

Although this review reports on chemical communication in BZ oscillating networks engineered using microfluidics, it is important also to highlight some results dealing with $B Z$ and compartmentalization obtained by other approaches than microfluidics. Okano et al. $^{44,}{ }^{48}$ studied the phase synchronization of a 2D array of $B Z$ cell elements in a gel matrix mediated by external illumination. To model neurotransmission signalling, which is typically unidirectional, and occurs via discrete pulses of mass exchange in contrast to continuous mass diffusion, Horvath et al. ${ }^{60}$ performed experiments with pulse-coupled BZ oscillators. The coupling strength between two oscillators was controlled by varying the concentration of the inhibitor specie bromide in one oscillator as a function of the oscillatory signal in the other. A similar method was employed by Weiner et al., ${ }^{61} \mathrm{Holz}$ et al. ${ }^{62}$ and Fukuda et al., ${ }^{41,42}$ conducting experiments and numerical simulations on the pacemaker entrainment of a 1D arrangement of ion-exchange beads loaded with a BZ catalyst, and submerged in a catalyst free BZ solution. Resin beads were also employed in a stirred bulk solution by Taylor et al. ${ }^{9,63}$ In these papers the oscillating behaviour was studied as a function of stirring rate and bead density.

In most of "chemical communication" experiments, it was shown that weak coupling, i.e. inhibitory coupling, usually generate desynchronized oscillators, and conversely for strong excitatory coupling. Similar results were also obtained with hydrogels ${ }^{64}$ and micrometre sized inorganic clay particles. ${ }^{65}$

\section{BZ compartmentalization using microfluidics:}

To fabricate bio-mimicking microreactors, microfluidics is an excellent choice. Up to now, microfluidic platforms have been generally used for the high through-put screening of chemical reactions, and for the compartmentalization of materials in libraries of isolated chemical micro-reactors. ${ }^{66,67}$ In particular, droplet-based microfluidics has been demonstrated as a powerful tool for encapsulating biological entities and chemical reagents in artificial monodisperse microcompartments, mostly constituted by water-in-oil microdroplets. ${ }^{68-71}$ In the context of chemical communication, this versatile technique is particularly useful to generate reproducible geometrically homogeneous systems of droplets, $^{72}$ emulsions, ${ }^{73}$ liposomes ${ }^{50}$ and polymersomes ${ }^{55,74}$ of well-defined size, dispersity and chemical composition, all of them acting as microreactors. The chemical communication between such microreactors can be obtained in various modalities: Either within the encapsulated entities, between the interior and the outside of the compartment (partition), or between neighbouring compartments. At the level of communication between neighbouring compartments the generation of intermediates that allow for the spatio-temporal propagation of chemical information is responsible for the production of collective behaviours, which can lead to coupling and synchronization. The spatio-temporal coupling between compartments can also be modulated and tuned using microfluidics, which provides not only a control over the dimensions and the boundaries of the compartments but also over their spatial organization and arrangements into different geometries including 1D and 2D networks. High control over the spatial resolution in manufacturing microfluidics 75,76 enables for engineering assemblies of microcompartments of different dimensions, where the characteristic time of diffusion between these chemically coupled entities is less than the actual time of reaction, e.g., the oscillatory periodicity of the encapsulated BZ-reaction. Under these conditions, the collective behaviour of coupled microreactors is governed by the diffusive pathway between the microreactors, and can hence be tuned by engineering permeable-specific membranes encapsulating the microreactors, or introducing scavengers in the diffusive medium.

One of the first attempts to use microfluidics techniques in connection with the BZ reaction was made by the group of Steinbock, ${ }^{77}$ who fabricated a patterned microfluidic device where BZ containing chambers were connected via a microchannel (see Figure 2), to constrain propagating wave signals in a well-defined path. By avoiding the typical wave broadening due to diffusion, with this system, they could 
improve the study of spiral segmentation and pattern formation in heterogeneous media.

An extensive general review on the potentiality of "active" droplets outside of equilibrium, from nano- to millimetre sized scale, has been recently published. ${ }^{78}$ In this review, we focus on a biomimetic model of chemical communication proceeding via a reaction/diffusion pathway. In the following we describe different microfluidic methodologies that have been designed to elaborate biomimetic networks of BZ micro-oscillators using microfluidic platforms and we present a summary of the corresponding results. As the main theme of this review is the generation of networks of BZ microoscillators using microfluidics, we shall focus on the multi-scale approach used for the assembly of networks ranging from 1D to 2D.

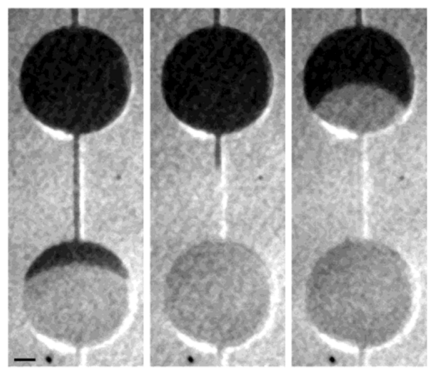

Figure 2. Oxidation wave traveling through a BZ system in a patterned microreactor The channel has a height of $80 \mu \mathrm{m}$ and a width of $50 \mu \mathrm{m}$. The circular portion of the reactor has a diameter of $1 \mathrm{~mm}$, and the length of the reference bar (A) is $200 \mu \mathrm{m}$. Initial $\mathrm{BZ}$ concentrations are $1.50 \mathrm{M} \mathrm{H}_{2} \mathrm{SO}_{4}, 0.15 \mathrm{M} \mathrm{1,4}$ cyclohexanedione, $0.10 \mathrm{M}$ $\mathrm{NaBrO}_{3}$, and $3.1 \mathrm{mM}$ ferroin. The snapshots are separated by $10 \mathrm{~s}$. Reprinted from ref. $^{77}$. Copyright $\odot 2004$, American Chemical Society.

BZ-oscillators stabilized by surfactant monolayers: from linear to planar networks

\section{1-D arrays of $B Z$}

A major contribution to the study of coupled compartmentalized oscillators came from the group of Epstein at Brandeis University. ${ }^{43}$ They used, for the first time, a Tshaped microfluidic junction (Figure $3 A$ ) to generate BZ containing aqueous droplets separated by octane and stabilized by a surfactant. Droplets formed at the junction were collected in glass capillaries with diameters of 100-200 $\mu \mathrm{m}$, which was rendered hydrophobic in order to obtain a linear network of BZ droplets. The authors showed the importance of the geometric configuration of the BZ containing droplets to control the dynamic behaviour of the network. For example coupling between droplets was only observed when the time of diffusion via the droplet spacing medium (which can be manipulated by the flow rate of the oil phase) was shorter than the period of the BZ oscillations. The oscillation dynamics was found to be rather sensitive to the concentration of the BZ component malonic acid (MA); at higher concentrations, anti-phase oscillation between neighbouring droplets was observed. For intermediate and smaller concentrations of $\mathrm{MA}$, the system reached stationary Turing patterns, occurring after a few anti-phase oscillation periods or immediately, respectively.

For these droplets, separated by a hydrophobic medium, nonpolar intermediates were thought to be responsible for the communication between the micro-compartments. In particular, the inhibitor $\mathrm{Br}_{2}$ had the proper characteristic to diffuse fast in the nonpolar medium and facilitate the communication between two adjacent oscillators. The experimental findings were also in line with an inhibitory coupling type, as theoretically predicted few years earlier. ${ }^{79}$ This was further confirmed by adding a scavenger for bromine to the organic phase; in this case the communication ceased to occur and the droplets oscillated eventually independently. Analyses of the collective behaviour of the networks of oscillating droplets, generally based on space-time plots constructed from time-series of images of the droplet recorded over times, as shown in Figure $3 \mathrm{~B}$ along with a simulation based on the FKN-model, led the authors to conclude that for the experimental conditions, the inhibitory coupling governed by bromine was dominant. In order to investigate these findings on a more quantitative basis by defining both the boundary and the initial conditions, Delgado et al. $^{49}$ exploited the photosensitivity of the catalyst $\mathrm{Ru}$ (bipy) ${ }_{3}{ }^{2+}$. The production of bromide within the individual drops could be hence controlled, and hereby inhibiting on demand the oscillations within the drops exposed to light. After the removal of the illumination, the drops could recover their ability to oscillate synchronously, achieving an initial phase synchronization of an entire array of oscillators. Arrays of BZ micro oscillators in a perfluorinated oil (HFE 7500) containing the amphiphilic block copolymer PEG-PFPE were generated using the flow focusing microfluidic device schematized in Figure $3 \mathrm{C}$ and parked inside $100 \mu \mathrm{m}$ diameter hydrophobically modified round glass capillary for manipulation and observation. Experimentally it was evidenced that such a drop with high bromide concentration positioned in the center of a 1D array of in-phase oscillators, emitted, upon ceasing illumination, a sufficient amount of bromide to cause neighbour drops to display out-of-phase coupling. Eventually, this caused the fraction of in-phase drops to decay monotonically, resulting in the entire array to oscillate out-of-phase. The perturbation of the in-phase oscillation was furthermore found to depend on the initial concentration of MA; higher concentrations resulted in weaker coupling and thus prolonging the time to attain anti-phase synchrony with a phase shift of exactly $180^{\circ}$. Numerical simulations based on an open system, i.e., the drops surveyed being positioned far from the boundaries, confirmed these findings. Restricting the number of oscillating drops by applying boundary conditions set by non-oscillating drops, and setting the surveyed drops in an initial synchronized state by illumination, the authors eventually observed the same general out-of-phase behaviour. However in this case, due to the restrictions introduced by the boundary conditions, the 
out-of-phase behaviour deviated slightly from the ideal $180^{\circ}$ anti-phase synchrony.

(A)
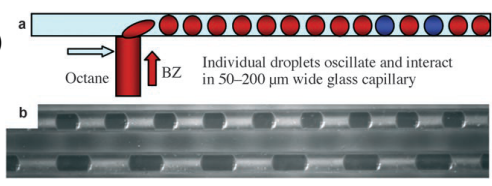

(B)

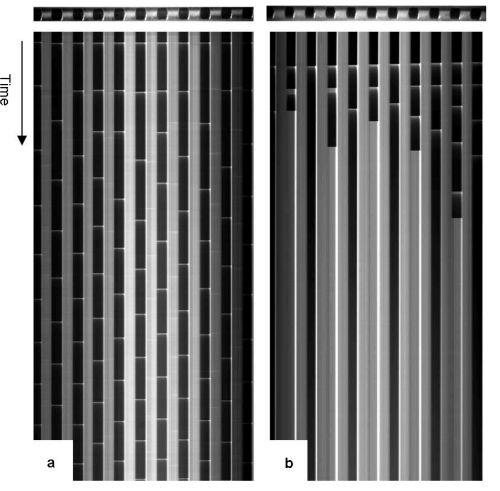

(C)

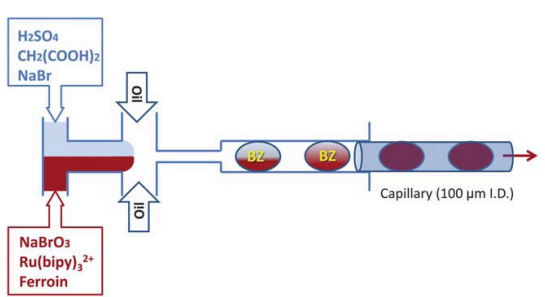

Figure 3. (A) Schematic representation of the microfluidic device. Red droplets correspond to the reduced form of the catalyst (ferroin), blue droplets to the droplets with convex surfaces are dark due to ferroin. Horizontal length of the frame and inner diameter (ID) of the capillary are $4.8 \mathrm{~mm}$ and $150 \mathrm{~mm}$ respectively. Reproduced from ref. ${ }^{43}$ Copyright (C) 2008 Wiley-VCH Verlag GmbH $\&$ Co. KGaA. Reproduced with permission. (B) Examples of space-time plots obtained by stacking consecutive images of the droplet arrays shown top-most in each image. (a) Anti-phase oscillations with spikes of oxidation of ferroin seen as light horizontal lines across BZ droplets and (b) stationary Turing structures with alternating oxidized and reduced states evolving from an initial oscillatory state. rorizontal lengths of the frame and the capillary are 4.8 mm and 150 mm, Patterns extend to the left and right of the segments shown. Patterns extend to the left and right of the segments shown. Reproduced from drawing of the microfluidic PDMS device drop generator. At left, two different aqueous streams containing complementary reactants of the BZ solution are injected into the drop generator (light blue and red). The streams merge and coflow down a central channel, meeting two perpendicular oil flows that generate BZ droplets in a nozzle. The co-flow of the BZ solution is preserved immediately after the drops are formed, but complete mixing takes place in less than $1 \mathrm{~s}$. A glass capillary (100 $\mathrm{mm}$ ID) was inserted in the PDMS chip a few millimetres downstream from the nozzle to collect the BZ droplet

The role of the $B Z$ intermediate bromine as the main messenger specie in the inhibitory coupling between BZ drops was also confirmed by $\mathrm{Li}$ et $a l^{51}$ who used the same microfluidic methodology. When the initial bromate concentration in the drops was increased, and the MA concentration was decreased, excitatory coupling leading to in-phase synchrony between neighbour drops was observed. Thus, under these conditions, the inter-droplet diffusion of the excitatory intermediate bromate dioxide radical plays a significant role. Based on finite element analysis, the authors suggested a theoretical model for the coupling strength between the $\mathrm{BZ}$ drops, as the ratio between the diffusive flux and the consumption of the inhibitor specie bromine. The transition from weak to strong coupling transforms the dynamical phase behaviour from out-of-phase to in-phase synchrony.

The possibility of cross-diffusion, i.e., the diffusion of one specie induced by a concentration gradient of another, has been investigated for other BZ species. ${ }^{47,80}$ In a microemulsion, cross-diffusion coefficients were established for various species like bromine, bromide, ferroin, bromate, etc. ${ }^{47,} 80,81$ These results suggest that a more complex diffusion-reaction pattern is occurring in the communication pathway between BZ droplets (see also Vanag et al. ${ }^{82}$ for a complete overview on the effects of cross-diffusion on pattern formation). Moreover, cross-diffusion can also generate hydrodynamic motions, if the dimensions of the reactor allow for bulk movements, thus potentially affecting the information delivery among single oscillators. ${ }^{83-85}$

(A)

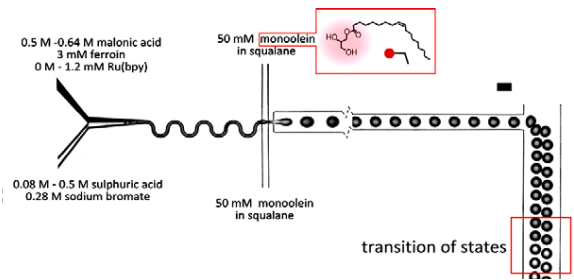

(B)
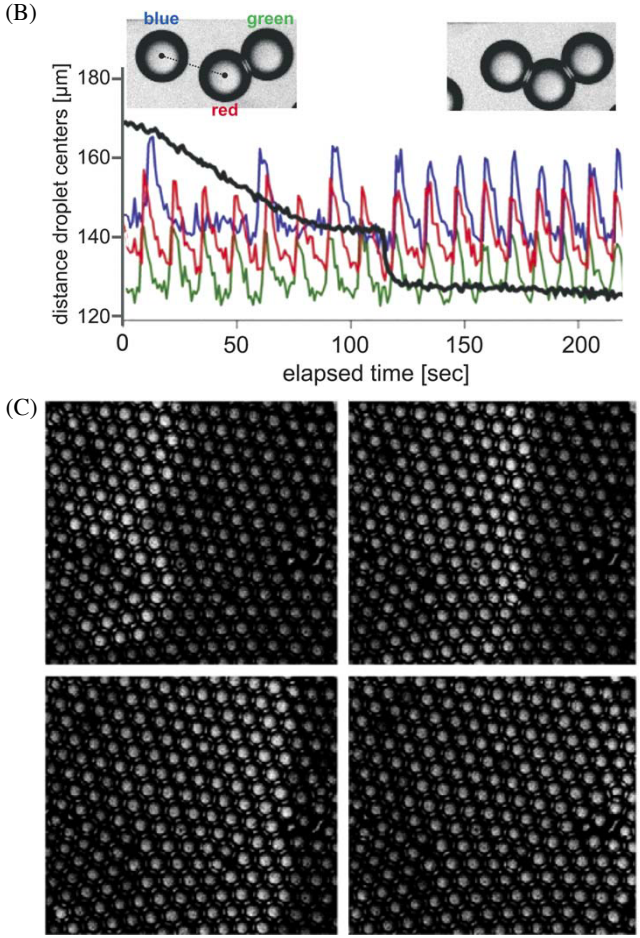

Figure 4. (A) The contents of the BZ reaction are mixed on a microfluidic chip to prevent any pre-reaction. Seen through an optical $480 / 20 \mathrm{~nm}$ notch filter, the transition from red colour to the blue colour of the $B Z$ reaction is seen by the change in the brightness of the droplet. Correspondingly, red appears dark/black and blue appears bright/white in the grayscale images. (Scale bar: $100 \mu \mathrm{m}$.). Reprinted from ref. ${ }^{86}$ with kind permission of The European Physical Journal
(EPJ). (B) Effect of membrane formation upon the phase coupling of chemical (EPJ). (B) Effect of membrane formation upon the phase coupling of chemica
osciliators. The blue, red, and green traces represent the transmittance of the three droplets shown in the insets as a function of time. The black curve shows on the same time axis, the distance of the "blue' from the "red" drople (measured center-to-center). Clearly, the oscillations couple in phase as soon as from ref 4 rane is formed (jump in the black curve), but not before. Reproduced of travelling waves when bilayer membranes are formed between oscillator droplets. Each image is $5 \mathrm{~s}$ apart. The droplet diameter is $30 \mu \mathrm{m}$. Reprinted from ref. ${ }^{66}$ with kind permission of The European Physical Journal (EPJ). 
In the previous examples, the BZ droplets were separated by a hydrophobic phase, and most frequently bromine was found to be the main messenger molecule diffusing between the microreactors promoting inhibitory coupling. What dynamical behaviour could result if BZ microreactors coated with a surfactant (present in the oil phase) were brought into close contact without coalescence? At the point of contact between two or more adjacent microreactors, as usually hypothesized a bilayer would be formed. This bilayer could give rise to new dynamic phenomena due to the interplay between the droplet network topologies and the type of coupling between the individual droplets. It was expected that mass transfer of less hydrophobic molecules, such as the BZ activator bromine dioxide radical, between the micoreactors would be facilitated. Thutupalli et al. ${ }^{45,86}$ demonstrated this effect by generating, through a flow-focusing channel, BZ droplets in squalane containing the surfactant monoolein (Figure 4A). When drops were separated by even a small volume of oil, the coupling remained inhibitory, whereas drops in contact only separated by a monoolein bilayer exhibited excitatory in-phase synchrony, see Figure $4 \mathrm{~B}$ and travelling waves could be observed in $2 \mathrm{D}$ arrays (Figure $4 \mathrm{C}$ ).

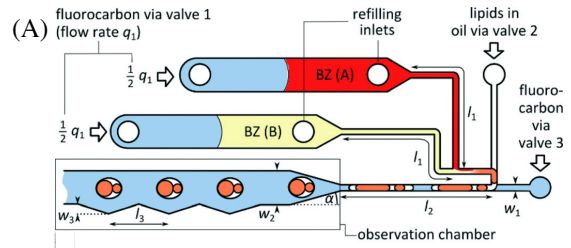

(B)

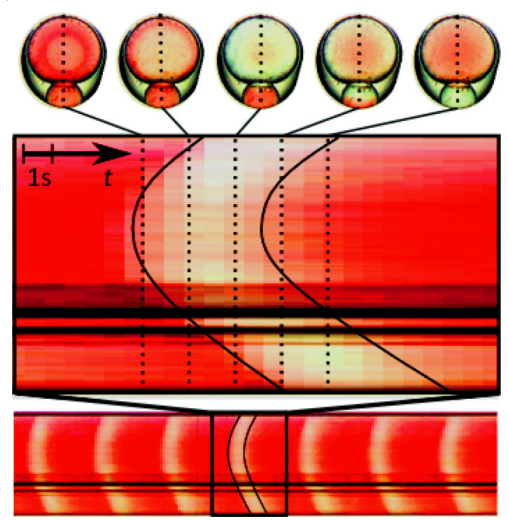

Figure 5. (A) Layout of channels on the microfluidic chip. The fluorocarbon phase supplied via valve 1 pushes BZ components $A$ and B. The 'refilling inlets' wer closed during the operation of the valves. The opening angle of the inlet to the observation chamber was $\alpha \approx 30^{\circ}$. This smooth widening prevented
fragmentation of the larger drops. The notches at the sidewall of the chamber served as traps for the capsules, allowing for their immobilization durin monitoring of the progress of reactions. The lengths of the channels were $11=1$ $\mathrm{mm}$ and $\mathrm{I} 2=9.5 \mathrm{~mm}$. The widths of the inlet and outlet channels at all three Tjunctions were equal to $\mathrm{w} 1=0.3 \mathrm{~mm}$ and their depths $\mathrm{h} 1=0.3 \mathrm{~mm}$. The width of the chamber was w2 $=2 \mathrm{~mm}$, the depth was h2 $=2 \mathrm{~mm}$, and the sizes of the notches were $\mathrm{w} 3=0.3 \mathrm{~mm}$ by $13=2 \mathrm{~mm}$. Reproduced from ref. with permission from the Royal Society of Chemistry. (B) Cuts of the snapshots of BZ-doublet along the dotted lines (top) collected on a single time-plot (middle and bottom). One can notice the parabolic shape of the white stripe representing the chemical wave of the oxidized catalyst expanding from the the center, the concentration of activator first exceeds the threshold for selfexcitation. The wave propagates outwards, passes through the facet between the droplets and propagates through the small drop. The long-time dynamics can be inferred from a staçk containing multiple sequences of oscillations (bottom) Reproduced from ref. ${ }^{33}$ with permission of the Royal Society of Chemistry.
So far, only networks of droplets with equal volumes have been considered. To extend the control over volumes to the level of individual compartments, Guzowski et al. ${ }^{53}$ described recently an automated microfluidic platform for reproducible self-assembly of chemically communicating droplet networks with pre-designed number and type of the communicating compartments. The chip was obtained by direct milling in polycarbonate and comprising a triple T-junction. To control the flow in the chip, and to generate the droplets on-demand, external valves were used. Pairs of BZ light sensitive droplets were encapsulated in an oil shell (hexadecane) containing asolectin (a mixture of phospholipids from soy lecitin) and dispersed in fluorocarbon oil, see Figure 5A. At the contact point in the core, a thin lipid membrane was also formed between the droplets. To immobilize the core shell droplets into 1D linear networks, their buoyancy were exploited to trap them in engraved notches. The authors demonstrated how resonance between chemical oscillators depended on the relative volumes of the droplets; smaller drops eventually became in-phase synchronized with the larger ones, by an excitatory coupling mechanism, see Figure 5B.

\section{From 1D to 2D networks}

In order to observe new dynamic regimes, and reach a higher level of complexity, a higher level of assembly geometry of the oscillators is necessary. For this purpose, microfluidics have much to offer, and more complex synchronization patterns were observed for higher order (1.5D and 2D) droplet configurations. Toiya et al. $^{46}$ obtained $1.5 \mathrm{D}$ networks of surfactant-coated drops loaded with BZ by using the same microfluidic device and conditions previously described. ${ }^{49}$ When the droplet diameter lied between $0.5 d$ and $d$, where $d$ is the diameter of the glass capillary, the droplet self-organized to form a zig-zag structure. To obtain 2D geometry, drops produced in the microfluidic device were transferred to a standard microscope slide. A variety of synchronous regimes were observed, including in- and anti-phase oscillations, stationary Turing patterns and more complex mixed patterns, including three-phase regimes.

The original Turing mechanism for morphogenesis ${ }^{87}$ was tested by Tompkins et al., ${ }^{23}$ who employed 1- and 2D arrays of $\mathrm{BZ}$ drops suspended in oil. By tuning the coupling strength via drop size and MA concentration, the authors were able to generate the spatiotemporal chemical structures predicted by Turing. Due to the heterogeneity of the experimental setup, i.e., the diffusion of trigger molecules between $\mathrm{BZ}$ drops occurs through an oil phase, in contrast to the homogeneously coupled cells employed in Turing's prediction, an additional structure was found. In the continuity of the work of Toiya et al., ${ }^{43}$ Delgado et al. $^{49}$ and Li et al., ${ }^{51}$ where the emulsion systems suffered from limited abilities to define custom network geometries and controlled boundary conditions, Tompkins et al. $^{52}$ described the ability to create planar (close packed spheres) networks with Dirichlet (constant concentration) boundary conditions. This was obtained by physical trapping by confining photosensitive BZ drops generated using the flow focusing device between two glass 
walls, and by optically isolating a subset of the drops using programmable illumination. In order to create geometries not found within close-packed spheres, they developed new microfluidic manufacturing techniques capable of constructing custom planar geometries in PDMS. PDMS is highly permeable to nonpolar fluids and gases, but relatively impermeable to polar and charged species. To obtain networks with Neumann boundary conditions (no-flux boundary), containment patterns were obtained by Deep Reactive Ion Etching (DRIE) into silicon wafers and forcing the BZ emulsion into the cavities under a glass sheet (Figure 6A). The differences between the observed network dynamics for each boundary condition were described (example Figure 6B). Using light, the authors demonstrated the ability to control the initial conditions of the network and to cause individual oscillators within the network to undergo sustained period elongation or a one-time phase delay.

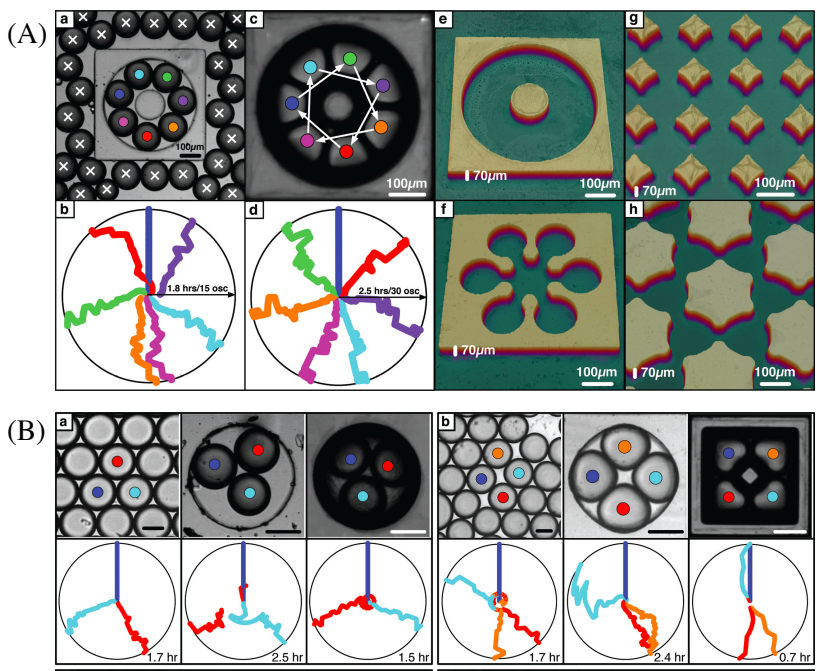

Figure 6. (A) Planar networks with custom geometries. Typical results shown. (a) A polydimethylsiloxane (PDMS) device designed to create a ring network of seven with constant-concentration boundary conditions. The drops color coded
as in (b). (b) A radial-phase-time plot of the ring of seven shown in (a). (c) An as in (b). (b) A radial-phase-time plot of the ring of seven shown in (a). (c) An etched silicon device designed to create a ring network of seven with no-flux indicate the order of oscillation. (d) A radial-phase-time plot of the ring of seven indicate the order of oscillation. (d) A radial-phase-time plot of the ring of seven
shown in (c). (e)-(h) Network patterns etched into silicon wafers imaged using an optical profilometer. (e) The ring network, (f) a six-star network, (g) a square lattice, and (h) a triangular lattice. Reprinted with permission from ref. Copyright (C 2015, American Institute of Physics. (B) Network patterns observed from ring networks of three and four members with constant-concentration and no-flux boundary conditions. Top: Images of the networks with the oscillating drops colour coded as below. All scale bars are $100 \mu \mathrm{m}$. Bottom: Radial-phasetime plots of the networks above. Left: Circular networks with constantconcentration boundary conditions implemented via optical isolation in closepacked drops. Only the colour coded drops are oscillatory; the other drops are optically inhibited. Middle: Circular networks with constant-concentration surrounding channels. The surrounding channels are not shown. Right: Circular networks with no-flux boundary conditions implemented in etched silicon. Reprinted with permission from ref. ${ }^{2}$ Copyright(C) 2015, American Institute of Physics.

As one of the main goals of building oscillatory networks is to approach biomimetic systems such as cells, and investigate their chemical communication, BZ droplets stabilized by 1,2dimyristoyl-sn-glycero-3-phosphocholine (DMPC) phospholipid layers at the interface, were generated using a coaxial flow microfluidic device by Rossi et al. ${ }^{54}$ The BZ droplets in the oil phase (chloroform:cyclohexane), the number of BZ drops and their network geometry, was controlled by sliding the point of confluence between the exit of the microfluidic device, and a viscous aqueous solution of polymer (polyvinyl alcohol). The results showed how chemical coupling of BZ microemulsions, locally separated by a phospholipid layer, eventually transformed an initial out-of-phase synchrony into excitatory in-phase synchrony via trans-bilayer diffusion of bromous acid.

\section{BZ-oscillators stabilized by surfactant bilayers:}

The water in oil compartments previously reported are, despite the biomimetic approach, still far from water in water cell-like compartments found in nature. Giant phospholipid vesicles (or liposomes), where water droplets are compartmentalized by phospholipid membranes and dispersed in a water medium, provide a more reliable and promising artificial cell-like platform. 88 With such, not only the mode of encapsulation, but also the permeability properties of the membrane and the inter-liposomes chemical communication will differ.

Tomasi et al. ${ }^{50}$ described, for the first time, the encapsulation of the BZ-reaction in liposomes, by employing a flow focusing microfluidic device (see figure 7A). This novel system provided the opportunity to study chemical communication between cell-like structures composed of a fDMPCt membrane, in a purely aqueous environment (see Figure 7B). This implied a high extent of partitioning of all the BZ species, some of them capable of crossing the phospholipid membrane, thus allowing for exploring the chemical communication pathways in a different environment with respect to the water-in-oil microdroplets. In this case, the microfluidic technique granted the best experimental method to obtain giant $(100-300 \mu \mathrm{m})$ liposomes in the presence of the acidic and oxidizing medium of the $\mathrm{BZ}$ reaction. Other techniques of liposome formation, such as the droplet transfer method, ${ }^{89,}{ }^{90}$ were effective for the formation of millimetre sized water-in-oil emulsions, but failed when droplets were dispersed in an aqueous medium. By using electrochemical techniques, it was possible to characterize the behaviour of the oxy-bromine intermediates at the interface between a hydrophilic and a hydrophobic medium, both in the presence and in the absence of a lipid membrane. ${ }^{50,91}$ The main outcome of this study was the unambiguous identification of brominated species as the messenger molecules between different compartments; this, together with numerical simulations, allowed to assess the activatorytype coupling, which was ensured by oxidation waves at the contact regions between different liposomes. A typical pulse transmission is depicted in Figure 7B. The liposomes generated by means of microfluidic techniques represent an interesting system to study the influence of the membranes on the communication dynamics in the network of chemical oscillators. In fact, to control the chemical communication, the membranes could be engineered to be selectively permeable to specific messenger molecules. ${ }^{92}$ In practice, by intercalating different dopants into the DMPC lipid bilayers, it was possible to tune some of the membranes properties, such as lamellarity, permeability and local curvature. The specific effect on the membrane structure of dopants like cholesterol, myristic acid, sodium tetradecyl sulphate and tetradecylamine was studied by small angle X-ray scattering (SAXS), and the 
role of each dopant on the oscillatory dynamics was investigated for micro-sized water/lipid/oil simple emulsions. ${ }^{93}$ The introduction of these bilayer intercalating species brought about new dynamical regimes with respect to the pure DMPC membranes, where, for simple emulsions, it was found a mostly inhibitory-type coupling. It was found, that the bromine-scavenger cholesterol induced the formation of weakly phase-coupled patterns, while the anionic surfactant sodium dodecyl sulphate, promoted a strongly coupled system with the presence of phase-lock states between alternate oscillators.

(A)

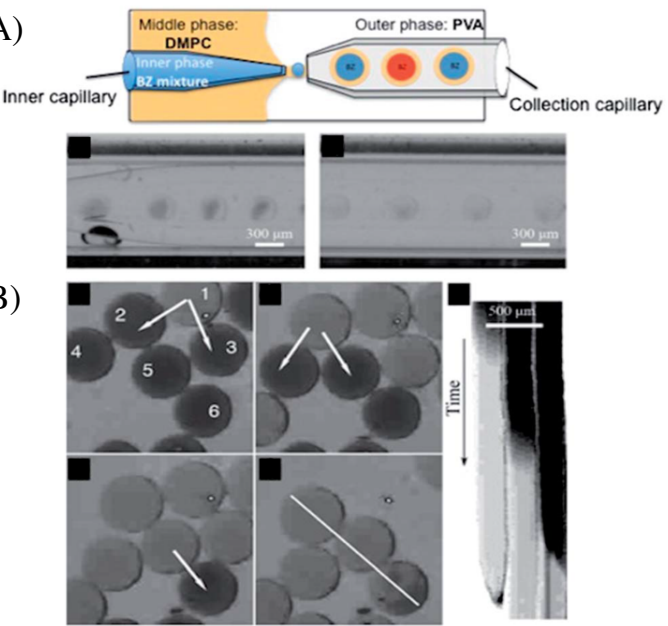

Figure 7. (A) Cartoon showing the coaxial microfluidic device and schematizing the encapsulation process. (B) Formation of the $B Z / O / W$ double emulsions in the microfluidic device at the entrance (below left) of the collection capillary and (below right) at the exit of the collection capillary, time elapsed $80 \mathrm{~s}$. (B) The pulse transmission across the touching liposomes after solvent evaporation. White arrows indicate the direction of pulse propagation. (Left): Image showing the space-time plot of liposomes 2, 5, 6 along the white bar. (B) Consecutive images showing a wave front propagating through three liposomes, along with the corresponding space-time plot. Reproduced from ref. ${ }^{50}$ with permission of the Royal Society of Chemistry.

To the best of our knowledge, this is the only example of chemical communication between vesicles encapsulating the BZ oscillator. However, it is important to mention that the successful entrapment of the BZ reaction inside giant polymersomes was recently reported by Mercader et al. ${ }^{55,56}$ These systems can offer new possibilities to study the influence of the membrane composition on the global behaviour in networks of chemical oscillators.

\section{Conclusions}

\section{Notes and references}

1. S. H. Strogatz, Sync: How Order Emerges From Chaos In the Universe, Nature, and Daily Life Hachette Books; Reprint edition (April 14, 2004), 2004.
Confined domains of the oscillatory Belousov-Zhabotinsky reaction arranged in various geometries, using microfluidics, have proven to be a significant and versatile tool for modelling bio-physical networks and communication pathways. The pertinent chemical species responsible for communication in these systems are, mainly, the inhibitory bromine and the excitatory bromine dioxide radical. However, other species, such as $\mathrm{Br}^{-}$and $\mathrm{HBrO}_{2}$ can play an important role. Since their concentrations are governed by the experimental conditions, various coupling patterns can be achievable, i.e., the phase shift between different oscillators is tuneable. In this respect, membranes and surfactants confining the microreactors also play a significant role. In particular, they can promote preferential communication pathways or act as specific scavengers for messenger molecules, thus allowing a finetuning of the general dynamical properties of the networks of chemical oscillators. Droplet arrays separated by an organic medium represent a valuable tool for investigating the motion of chemical species through long distances, compared to the molecular scale, and for engineering coupled aqueous domains. Importantly, both the geometry and structure of compartmentalized oscillators can be finely tuned using microfluidic devices. The applications of such systems are not entirely defined yet, though they are considered as prototypes for many innovative and technically sophisticated devices. Clearly, the most challenging configurations are those in which both the internal and external compartments (where signal emitters are produced and exchanged) encompass an aqueous medium. Semipermeable membranes, surrounding oil or water droplets, also represent a boundary region whose properties are still being investigated. In general, due to their "soft" nature, the means to study compartments separated by mono or multishell amphiphilic membranes, without perturbations, are limited and often indirect. Thus, only a broad range of physico-chemical, biological and computational methods can afford extensive understanding of communication within networks of chemically coupled oscillators confined in liquid domains. Microfluidics is a versatile platform, and offers exceptional technical control, for the production and organization of liquid droplets into different geometries. However, up to now all the geometries reported are 1D or 2D, with fixed positions of the droplets. Elaborating active geometries with different dimensions (1D to 3D), and ondemand oscillating networks would overwhelm the field of network dynamics, with new emergent patterns, new collective properties and advance of our knowledge on chemical communication.

2. I. Z. Kiss and J. L. Hudson, Aiche Journal, 2003, 49, 22342241.

3. I. R. Epstein and B. Xu, Nat Nano, 2016, 11, 312-319.

4. K. Showalter and I. R. Epstein, Chaos, 2015, 25, 097613.

5. G. Ghoshal, A. P. Muñuzuri and J. Pérez-Mercader, Scientific Reports, 2016, 6, 19186. 
6. B. N. Kholodenko, Nature reviews. Molecular cell biology, 2006, 7, 165-176.

7. James E. Ferrell, Jr., Tony Y.-C. Tsai and Q. Yang, Cell, 144, 874-885.

8. S. H. Strogatz, Sync: The Emerging Science of Spontaneous Order Penguin, New Ed (29 avril 2004).

9. A. F. Taylor, M. R. Tinsley, F. Wang, Z. Huang and K. Showalter, Science, 2009, 323, 614-617.

10. I. R. Epstein, Chemical Communications, 2014, 50, 1075810767.

11. M. W. Barnett and P. M. Larkman, Practical Neurology, 2007, 7, 192-197.

12. Y. Arai, T. Shibata, S. Matsuoka, M. J. Sato, T. Yanagida and M. Ueda, Proceedings of the National Academy of Sciences, 2010, 107, 12399-12404.

13. J. Holley, A. Adamatzky, L. Bull, B. De Lacy Costello and I. Jahan, Nano Communication Networks, 2011, 2, 50-61.

14. A. Adamatzky, J. Holley, P. Dittrich, J. Gorecki, B. De Lacy Costello, K.-P. Zauner and L. Bull, Biosystems, 2012, 109, 7277.

15. P. L. Gentili, RSC Adv., 2013, 3, 25523-25549.

16. Advances in Artificial Life, Evolutionary Computation and Systems Chemistry, Springer International Publishing, Cham, 2016.

17. P. H. King, J. C. Corsi, B.-H. Pan, H. Morgan, M. R. R. de Planque and K.-P. Zauner, Biosystems, 2012, 109, 18-23.

18. B. P. Belousov, A periodic reaction and its mechanism", Sbornik Referatov po Radiatsonno Meditsine, Moscow, 1958.

19. A. M. Zhabotinsky, Proc. Acad. Sci. USSR, , 1964, 157, 392395.

20. J. Higgins, Proceedings of the National Academy of Sciences of the United States of America, 1964, 51, 989-994.

21. B. Novak and J. J. Tyson, Nat Rev Mol Cell Biol, 2008, 9, 981991.

22. S. N. Semenov, A. S. Y. Wong, R. M. van der Made, S. G. J. Postma, J. Groen, H. W. H. van Roekel, T. F. A. de Greef and W. T. S. Huck, Nat Chem, 2015, 7, 160-165.

23. N. Tompkins, N. Li, C. Girabawe, M. Heymann, G. B. Ermentrout, I. R. Epstein and S. Fraden, PNAS, 2014, 111, 4397-4402.

24. K. Bar-Eli, The Journal of Physical Chemistry, 1984, 88, 36163622.

25. M. Rosenblum and A. Pikovsky, Contemporary Physics, 2003, 44, 401-416.

26. Y. Kuramoto, Physica A: Statistical Mechanics and its Applications, 1981, 106, 128-143.

27. M. N. Stolyarov, V. A. Romanov and E. I. Volkov, Physical Review E, 1996, 54, 163-169.

28. M. Dolnik and I. R. Epstein, Physical Review E, 1996, 54, 3361-3368.

29. Y. Kuramoto, Chemical oscillations, waves, and turbulence, Springer Science \& Business Media, 2012.

30. A. M. Zhabotinsky and F. Rossi, International Journal of Design \& Nature and Ecodynamics, 2006, 1, 323-326.

31. N. Marchettini, M. Antonio Budroni, F. Rossi, M. Masia, M. Liria Turco Liveri and M. Rustici, Physical Chemistry Chemical Physics, 2010, 12, 11062-11069.
32. A. F. Taylor, Progress in Reaction Kinetics and Mechanism, 2002, 27, 247-325.

33. V. K. Vanag and I. R. Epstein, Physical Review Letters, 2001, 87, 228301.

34. F. Rossi, S. Ristori, M. Rustici, N. Marchettini and E. Tiezzi, Journal of Theoretical Biology, 2008, 255, 404-412.

35. R. J. Field, Journal of Chemical Education, 2000, 77, 450.

36. I. R. Epstein and K. Showalter, The Journal of Physical Chemistry, 1996, 100, 13132-13147.

37. L. Sciascia, F. Rossi, C. Sbriziolo, M. L. T. Liveri and R. Varsalona, Physical Chemistry Chemical Physics, 2010, 12, 11674-11682.

38. J. J. Tyson, Frontiers in Mathematical Biology, 1994, 100, 569-587.

39. R. J. Field, E. Koros and R. M. Noyes, Journal of the American Chemical Society, 1972, 94, 8649-8664.

40. R. Toth and A. F. Taylor, Progress in Reaction Kinetics and Mechanism, 2006, 31, 59-115.

41. H. Fukuda, N. Tamari, H. Morimura and S. Kai, The Journal of Physical Chemistry A, 2005, 109, 11250-11254.

42. H. Fukuda, H. Morimura and S. Kai, Physica D: Nonlinear Phenomena, 2005, 205, 80-86.

43. M. Toiya, V. K. Vanag and I. R. Epstein, Angewandte Chemie International Edition, 2008, 47, 7753-7755.

44. T. Okano and K. Miyakawa, Physical Review E, 2009, 80, 026215.

45. S. Thutupalli, S. Herminghaus and R. Seemann, Soft Matter, 2011, 7, 1312-1320.

46. M. Toiya, H. O. González-Ochoa, V. K. Vanag, S. Fraden and I. R. Epstein, The Journal of Physical Chemistry Letters, 2010, 1, 1241-1246.

47. F. Rossi, V. K. Vanag and I. R. Epstein, Chemistry - A European Journal, 2011, 17, 2138-2145.

48. T. Okano, A. Kitagawa and K. Miyakawa, Physical Review E, 2007, 76, 046201.

49. J. Delgado, N. Li, M. Leda, H. O. Gonzalez-Ochoa, S. Fraden and I. R. Epstein, Soft Matter, 2011, 7, 3155-3167.

50. R. Tomasi, J.-M. Noel, A. Zenati, S. Ristori, F. Rossi, V. Cabuil, F. Kanoufi and A. Abou-Hassan, Chem. Sci., 2014, 5, 18541859.

51. N. Li, J. Delgado, H. O. Gonzalez-Ochoa, I. R. Epstein and S. Fraden, Physical Chemistry Chemical Physics, 2014, 16, 10965-10978.

52. N. Tompkins, M. C. Cambria, A. L. Wang, M. Heymann and S. Fraden, Chaos, 2015, 25, 064611.

53. J. Guzowski, K. Gizynski, J. Gorecki and P. Garstecki, Lab on a Chip, 2016, 16, 764-772.

54. A. Z. F. Rossi, S. Ristori, J-M. Noel, V. Cabuil, F. Kanoufi and A. Abou-Hassan, Int. J. Unc. Comp., 2015, 11 (1), 23-36.

55. Y. Hu and J. Pérez-Mercader, Colloids and Surfaces B: Biointerfaces, 2016, 146, 406-414.

56. T. Pereira de Souza and J. Perez-Mercader, Chemical Communications, 2014, 50, 8970-8973.

57. A. N. Zaikin and A. M. Zhabotinsky, Nature, 1970, 225, 535537.

58. A. M. Zhabotinsky and A. N. Zaikin, Journal of Theoretical Biology, 1973, 40, 45-61. 
59. R. J. Field and M. Burger, Oscillations and Traveling Waves in Chemical Systems, John Wiley \& Sons, New York, 1985.

60. V. Horvath, P. L. Gentili, V. K. Vanag and I. R. Epstein, Angewandte Chemie International Edition, 2012, 51, 68786881.

61. J. Weiner, R. Holz, F. W. Schneider and K. Bar-Eli, The Journal of Physical Chemistry, 1992, 96, 8915-8919.

62. R. Holz and F. W. Schneider, The Journal of Physical Chemistry, 1993, 97, 12239-12243.

63. A. F. Taylor, M. R. Tinsley, F. Wang and K. Showalter, Angewandte Chemie, 2011, 123, 10343-10346.

64. M. L. Smith, K. Heitfeld, C. Slone and R. A. Vaia, Chemistry of Materials, 2012, 24, 3074-3080.

65. F. Rossi, S. Ristori, N. Marchettini and O. L. Pantani, J. Phys. Chem. C, 2014, 118, 24389-24396.

66. H. Song, D. L. Chen and R. F. Ismagilov, Angewandte Chemie International Edition, 2006, 45, 7336-7356.

67. C. Lu and S. S. Verbridge, Microfluidic Methods for Molecular Biology, Springer International Publishing, 1 edn., 2016.

68. N. Shembekar, C. Chaipan, R. Utharala and C. A. Merten, Lab on a Chip, 2016, 16, 1314-1331.

69. D. J. Collins, A. Neild, A. deMello, A.-Q. Liu and Y. Ai, Lab on $a$ Chip, 2015, 15, 3439-3459.

70. S.-Y. Teh, R. Lin, L.-H. Hung and A. P. Lee, Lab on a Chip, 2008, 8, 198-220.

71. M. T. Guo, A. Rotem, J. A. Heyman and D. A. Weitz, Lab on $a$ Chip, 2012, 12, 2146-2155.

72. G. F. Christopher and S. L. Anna, Journal of Physics D: Applied Physics, 2007, 40, R319.

73. M. Seo, C. Paquet, Z. Nie, S. Xu and E. Kumacheva, Soft Matter, 2007, 3, 986-992.

74. H. C. Shum, J.-W. Kim and D. A. Weitz, Journal of the American Chemical Society, 2008, 130, 9543-9549.

75. J. Friend and L. Yeo, Biomicrofluidics, 2010, 4, 026502.

76. C. Iliescu, H. Taylor, M. Avram, J. Miao and S. Franssila, Biomicrofluidics, 2012, 6, 016505.

77. B. T. Ginn, B. Steinbock, M. Kahveci and O. Steinbock, The Journal of Physical Chemistry A, 2004, 108, 1325-1332.

78. S. Lach, S. M. Yoon and B. A. Grzybowski, Chemical Society Reviews, 2016, DOI: 10.1039/C6CS00242K.

79. V. K. Vanag and I. R. Epstein, The Journal of Chemical Physics, 2003, 119, 7297-7307.

80. F. Rossi, V. K. Vanag, E. Tiezzi and I. R. Epstein, The Journal of Physical Chemistry B, 2010, 114, 8140-8146.

81. V. K. Vanag, F. Rossi, A. Cherkashin and I. R. Epstein, Journal of Physical Chemistry B, 2008, 112, 9058-9070.

82. V. K. Vanag and I. R. Epstein, Phys Chem Chem Phys, 2009, 11, 897-912.

83. M. A. Budroni, Phys Rev E, 2015, 92.

84. M. A. Budroni, L. Lemaigre, A. De Wit and F. Rossi, Physical Chemistry Chemical Physics, 2015, 17, 1593-1600.

85. M. A. Budroni, J. Carballido-Landeira, A. Intiso, A. D. Wit and F. Rossi, Chaos: An Interdisciplinary Journal of Nonlinear Science, 2015, 25, 064502.

86. S. Thutupalli and S. Herminghaus, The European Physical Journal E, 2013, 36, 1-10.
87. A. M. Turing, Philosophical Transactions of the Royal Society of London B: Biological Sciences, 1952, 237, 37-72.

88. G. Cevc, Phospholipids Handbook, Taylor and Franciss, 1994.

89. P. Stano, T. P. de Souza, P. Carrara, E. Altamura, E. D'Aguanno, M. Caputo, P. L. Luisi and F. Mavelli, Mech Adv Mater Struc, 2015, 22, 748-759.

90. S. Pautot, B. J. Frisken and D. A. Weitz, Langmuir, 2003, 19, 2870-2879.

91. T. J. Stockmann, J.-M. Noël, S. Ristori, C. Combellas, A. AbouHassan, F. Rossi and F. Kanoufi, Analytical Chemistry, 2015, 87, 9621-9630.

92. K. Torbensen, F. Rossi, O. L. Pantani, S. Ristori and A. AbouHassan, The Journal of Physical Chemistry B, 2015, 119, 10224-10230.

93. K. Torbensen, S. Ristori, F. Rossi and A. Abou-Hassan, Submitted, 2016. 